PROCEEDINGS OF THE

AMERICAN MATHEMATICAL SOCIETY

Volume 132, Number 9, Pages 2655-2660

S 0002-9939(04)07384-8

Article electronically published on March 24, 2004

\title{
MIXED-MEAN INEQUALITIES FOR SUBSETS
}

\author{
GANGSONG LENG, LIN SI, AND QINGSAN ZHU \\ (Communicated by Carmen C. Chicone)
}

\begin{abstract}
For $A \subset X=\left\{x_{1}, \ldots, x_{n} \mid x_{i} \geq 0, i=1,2, \ldots, n\right\}$, let $a_{A}$ and $g_{A}$ denote the arithmetic mean and geometric mean of elements of $A$, respectively. It is proved that if $k$ is an integer in $\left(\frac{n}{2}, n\right]$, then

$$
\left(\prod_{\substack{|A|=k \\ A \subset X}} a_{A}\right)^{\frac{1}{C_{n}^{k}}} \geq \frac{1}{C_{n}^{k}}\left(\sum_{\substack{|A|=k \\ \mid A \subset X}} g_{A}\right),
$$

with equality if and only if $x_{1}=\ldots=x_{n}$. Furthermore, as a generalization of this inequality, a mixed power-mean inequality for subsets is established.
\end{abstract}

\section{INTRODUCTION}

The classical arithmetic-geometric mean inequality is one of the most important analytic inequalities, and is used in almost every branch of mathematics. There is a huge amount of work on its generalization (see [1], [3]-7]).

In [2] (also see [6] 3.9.69]), Carlson established the following elegant mixed-mean inequality:

Let the arithmetic and geometric means of the real nonnegative numbers $x_{1}, \ldots, x_{n}$ taken $n-1$ at a time be denoted by

$$
a_{i}=\frac{x_{1}+\ldots+x_{n}-x_{i}}{n-1}, \quad g_{i}=\left(\frac{x_{1} \cdots x_{n}}{x_{i}}\right)^{\frac{1}{n-1}} .
$$

Then for $n \geq 3$,

$$
\left(a_{1} \cdots a_{n}\right)^{\frac{1}{n}} \geq \frac{g_{1}+\ldots+g_{n}}{n} .
$$

In this note, we establish a new mixed arithmetic-geometric mean inequality for subsets, which is an extension of the Carlson inequality and also an extension of the arithmetic-geometric mean inequality. Furthermore, we generalize our mixed arithmetic-geometric mean inequality for subsets to mixed power mean.

Received by the editors May 15, 2003 and, in revised form, June 9, 2003.

2000 Mathematics Subject Classification. Primary 26A51; Secondary 26B25, 26D07.

Key words and phrases. Mixed mean, power mean, Carlson inequality.

This work was supported partly by the National Natural Sciences Foundation of China (10271071). 
Our main results are the following two theorems.

Theorem 1. Let $X=\left\{x_{1}, \ldots, x_{n} \mid x_{i} \geq 0, i=1,2, \ldots, n\right\}$. For $A \subset X$, let $a_{A}$ and $g_{A}$ denote the arithmetic mean and geometric mean of all elements of $A$, respectively. If $k$ is an integer in $\left(\frac{n}{2}, n\right]$, then

$$
\left(\prod_{\substack{|A|=k \\ A \subset X}} a_{A}\right)^{\frac{1}{C_{n}^{k}}} \geq \frac{1}{C_{n}^{k}}\left(\sum_{\substack{|A|=k \\ A \subset X}} g_{A}\right),
$$

with equality if and only if $x_{1}=\ldots=x_{n}$.

Theorem 2. Let $X=\left\{x_{1}, \ldots, x_{n} \mid x_{i} \geq 0, i=1,2, \ldots, n\right\}$. For $A \subseteq X$ and $p \in R^{+}$, let $a_{p A}$ denote the $p$-th power mean of all elements of $A$, i.e.,

$$
a_{p A}=\left(\frac{\sum_{x_{i} \in A} x_{i}^{p}}{|A|}\right)^{\frac{1}{p}} .
$$

If $k$ is an integer in $\left(\frac{n}{2}, n\right]$ and $p>q>0$, then

$$
\left(\frac{\sum_{\substack{|A|=k \\ A \subset X}}\left(a_{p A}\right)^{q}}{C_{n}^{k}}\right)^{\frac{1}{q}} \geq\left(\frac{\sum_{A \subset X}^{|A|=k}\left(a_{q A}\right)^{p}}{C_{n}^{k}}\right)^{\frac{1}{p}},
$$

with equality if and only if $x_{1}=\ldots=x_{n}$.

Remark 1. Taking $k=n$ in Theorem 1, inequality (1) is just the arithmeticgeometric mean inequality. Taking $k=n$ in Theorem 2, inequality (2) is just the famous power-mean inequality. Taking $k=n-1$ in Theorem 1, inequality (1) is just the Carlson inequality. For $p=1$ and passing to the limit as $q \rightarrow 0$ in Theorem 2, we see that (2) implies (1). Hence, inequality (2) is a generalization of (1).

Remark 2. If $k \leq\left[\frac{n}{2}\right]$, taking $x_{1}=x_{2}=\ldots=x_{k}=l, x_{k+1}=\ldots=x_{n}=0$ in (1), then the left-hand side of (1) equals $l / C_{n}^{k}$, but the right-hand side is zero. Hence the statement of Theorem 1 fails for $k \leq\left[\frac{n}{2}\right]$.

\section{Proof of main Results}

Proof of Theorem 1. Put $I=\left\{1,2, \ldots, C_{n}^{k}\right\}$ and $\mathcal{X}_{k}=\{A \subset X|| A \mid=k\}$, and note that there is an injective mapping $f: I \longrightarrow \mathcal{X}_{k}$.

We will prove the following equality:

$$
a_{f(i)}=\frac{1}{C_{n}^{k}}\left(a_{f(i) \cap f(1)}+a_{f(i) \cap f(2)}+\ldots+a_{f(i) \cap f\left(C_{n}^{k}\right)}\right) .
$$

For this, let $f(i)=\left\{x_{i_{1}}, x_{i_{1}}, \ldots, x_{i_{k}}\right\}$, so that the left-hand side of (3) is equal to

$$
\frac{1}{k}\left(x_{i_{1}}+x_{i_{2}}+\ldots+x_{i_{k}}\right) \text {. }
$$

We may assume that the right-hand side of (3) can be written as

$$
b_{1} x_{i_{1}}+b_{2} x_{i_{2}}+\ldots+b_{k} x_{i_{k}},
$$

where $b_{i} \in R^{+}(i=1,2, \ldots, k)$.

Obviously, $x_{i_{j}}$ and $x_{i_{l}}(j, l \in\{1, \ldots, k\}, j \neq l)$ have the same coefficient. So it follows that

$$
b_{1}=b_{2}=\ldots=b_{k} .
$$


Since $k>\frac{n}{2}$, we have

$$
f(i) \cap f(j) \neq \Phi, \quad j=1,2, \ldots, C_{n}^{k} .
$$

Thus the sum of the coefficients of all elements of $a_{f(i) \cap f(j)}$ equals 1 ; hence

$$
b_{1}+b_{2}+\ldots+b_{k}=\frac{1}{C_{n}^{k}}(\underbrace{1+1+\ldots+1}_{C_{n}^{k}})=1 .
$$

From (4) and (5), we obtain

$$
b_{1}=b_{2}=\ldots=b_{k}=\frac{1}{k} .
$$

Thus (3) is proved.

Next, we will prove the following analogue to (3):

$$
g_{f(i)}=\left(\prod_{j=1}^{C_{n}^{k}} g_{f(i) \cap f(j)}\right)^{\frac{1}{C_{n}^{k}}} .
$$

By applying the arithmetic-geometric inequality, we get

$$
a_{f(i) \cap f(j)} \geq g_{f(i) \cap f(j)} .
$$

From (3) and (7), we infer that

$$
a_{f(i)} \geq \frac{1}{C_{n}^{k}} \sum_{j=1}^{C_{n}^{k}} g_{f(i) \cap f(j)} .
$$

Therefore,

$$
\begin{aligned}
\left(\prod_{\substack{|A|=k \\
A \subset X}} a_{A}\right)^{\frac{1}{C_{n}^{k}}} & =\left(\prod_{i=1}^{C_{n}^{k}} a_{f(i)}\right)^{\frac{1}{C_{n}^{k}}} \\
& \geq \frac{1}{C_{n}^{k}}\left(\prod_{i=1}^{C_{n}^{k}}\left(\sum_{j=1}^{C_{n}^{k}} g_{f(i) \cap f(j)}\right)\right)^{\frac{1}{C_{n}^{k}}} .
\end{aligned}
$$

On the other hand, using the discrete case of Hölder's inequality in the form

$$
\sum_{k=1}^{n}\left(\prod_{j=1}^{m} x_{j k}\right)^{\frac{1}{m}} \leq\left(\prod_{j=1}^{n}\left(\sum_{k=1}^{m} x_{j k}\right)\right)^{\frac{1}{m}},
$$

where $n, m \in N^{+}$and $x_{j k} \geq 0(j, k=1,2, \ldots, m)$ (see [6] 2.14.2]), we obtain

$$
\left(\prod_{i=1}^{C_{n}^{k}}\left(\sum_{j=1}^{C_{n}^{k}} g_{f(i) \cap f(j)}\right)\right)^{\frac{1}{C_{n}^{k}}} \geq \sum_{i=1}^{C_{n}^{k}}\left(\prod_{j=1}^{C_{n}^{k}} g_{f(i) \cap f(j)}\right)^{\frac{1}{C_{n}^{k}}} .
$$


Combining (6), (8) and (10), we see that

$$
\begin{aligned}
\left(\prod_{\substack{|A|=k \\
A \subset X}} a_{A}\right)^{\frac{1}{C_{n}^{k}}} & \geq \frac{1}{C_{n}^{k}} \sum_{i=1}^{C_{n}^{k}}\left(\prod_{j=1}^{C_{n}^{k}} g_{f(i) \cap f(j)}\right)^{\frac{1}{C_{n}^{k}}} \\
& =\frac{1}{C_{n}^{k}} \sum_{i=1}^{C_{n}^{k}} g_{f(i)}=\frac{1}{C_{n}^{k}} \sum_{\substack{|A|=k \\
A \subset X}} g_{A},
\end{aligned}
$$

which is just the inequality (1).

\section{Proof of Theorem 2.}

Lemma 1. Suppose that $\left(c_{i j}\right)$ is an $m \times n$ matrix with nonnegative entries. If $q<1$, then the $q$-th power mean of all the arithmetic means of the rows is greater than or equal to the arithmetic mean of all the q-th power means of the columns.

Proof. It suffices to prove the inequality

$$
\left(\frac{\sum_{i=1}^{m}\left(\frac{\sum_{j=1}^{n} c_{i j}}{n}\right)^{q}}{m}\right)^{\frac{1}{q}} \geq \frac{\sum_{j=1}^{n}\left(\frac{\sum_{i=1}^{m}\left(c_{i j}\right)^{q}}{m}\right)^{\frac{1}{q}}}{n},
$$

or equivalently,

$$
\left(\sum_{i=1}^{m}\left(\sum_{j=1}^{n} c_{i j}\right)^{q}\right)^{\frac{1}{q}} \geq \sum_{j=1}^{n}\left(\sum_{i=1}^{m}\left(c_{i j}\right)^{q}\right)^{\frac{1}{q}}
$$

which is just Minkowski's inequality.

We will prove the inequality (2) in two steps.

First, we prove that if (2) holds for $p=1$, then (2) holds for $p \in R^{+}$.

In fact, for $A=\left\{x_{i_{1}}, \ldots, x_{i_{k}}\right\} \subset X$, let $y_{i}=x_{i}{ }^{p}, \alpha=\frac{q}{p}, Y=\left\{y_{1}, \ldots, y_{n}\right\}$, and $A^{\prime}=\left\{y_{i_{1}}, \ldots, y_{i_{k}}\right\} \subset Y$. Then, the left-hand side of (2) equals

$$
\left(\frac{\sum_{\substack{|A|=k \\ A \subset X}}\left(\frac{\sum_{x_{i} \in A} x_{i}^{p}}{k}\right)^{\frac{q}{p}}}{C_{n}^{k}}\right)^{\frac{1}{q}}=\left(\frac{\sum_{\substack{\left|A^{\prime}\right|=k \\ A^{\prime} \subset Y}}\left(\frac{\sum_{y \in A^{\prime}} y}{k}\right)^{\alpha}}{C_{n}^{k}}\right)^{\frac{1}{q}},
$$

and the right-hand side of (2) is

$$
\left(\frac{\sum_{\substack{|A|=k \\ A \subset X}}\left(\frac{\sum_{x_{i} \in A} x_{i}^{q}}{k}\right)^{\frac{p}{q}}}{C_{n}^{k}}\right)^{\frac{1}{p}}=\left(\frac{\sum_{A^{\prime} \subset Y}\left(\frac{\sum_{y \in A^{\prime}} y^{\alpha}}{k}\right)^{\frac{1}{\alpha}}}{C_{n}^{k}}\right)^{\frac{1}{p}} .
$$

Hence, (2) is equivalent to the following inequality:

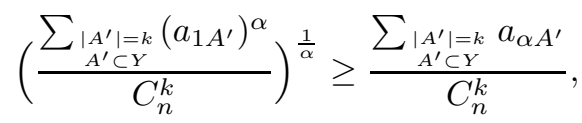

which is just inequality (2) for $p=1(q=\alpha<1)$.

Second, we prove that (2) holds when $p=1$.

In fact, let $C=\left(c_{i j}\right)$ be a $C_{n}^{k} \times C_{n}^{k}$ matrix with rows indexed by $\mathcal{X}_{k}=\{f(i) \mid f(i) \subset$ $X,|f(i)|=k\}$ and columns indexed also by $\mathcal{X}_{k}$, where

$$
c_{i j}=a_{q f(i) \cap f(j)} .
$$


The $q$-th power mean of the $j$-th column equals

$$
\begin{aligned}
\left(\frac{1}{C_{n}^{k}} \sum_{i=1}^{C_{n}^{k}}\left(a_{q f(i) \cap f(j)}\right)^{q}\right)^{\frac{1}{q}} & =\left(\frac{1}{C_{n}^{k}} \sum_{i=1}^{C_{n}^{k}}\left(\left(\sum_{x \in f(i) \cap f(j)} \frac{x^{q}}{|f(i) \cap f(j)|}\right)^{\frac{1}{q}}\right)^{q}\right)^{\frac{1}{q}} \\
& =\left(\frac{1}{C_{n}^{k}} \sum_{i=1}^{C_{n}^{k}} \sum_{x \in f(i) \cap f(j)} \frac{x^{q}}{|f(i) \cap f(j)|}\right)^{\frac{1}{q}} .
\end{aligned}
$$

Since the elements of $f(j)$ are in the same situation, the right-hand side of the above equality does not contain the term $x_{s}^{q}$ (if $x_{s} \notin f(j)$ ) and the sum of the coefficients of all $x_{t}^{q}$ (if $x_{t} \in f(j)$ ) equals 1 . Thus we have

$$
\left(\frac{1}{C_{n}^{k}} \sum_{i=1}^{C_{n}^{k}}\left(a_{q f(i) \cap f(j)}\right)^{q}\right)^{\frac{1}{q}}=\left(\frac{1}{k} \sum_{x \in g(j)} x^{q}\right)^{\frac{1}{q}} .
$$

Hence, the arithmetic mean of all the $q$-th power means of the columns is equal to

$$
\frac{1}{C_{n}^{k}} \sum_{j=1}^{C_{n}^{k}}\left(\frac{1}{k} \sum_{x \in g(j)} x^{q}\right)^{\frac{1}{q}}=\frac{1}{C_{n}^{k}} \sum_{\substack{|A|=k \\ A \subset X}} a_{q A} .
$$

On the other hand, according to the famous power mean inequality, and noting that $q<1$, we have

$$
c_{i j}=a_{q f(i) \cap f(j)} \leq a_{1 f(i) \cap f(j)} .
$$

It follows that the arithmetic mean of the $i$-th row is less than or equal to

$$
\begin{aligned}
\frac{1}{C_{n}^{k}} \sum_{j=1}^{C_{n}^{k}} a_{1 f(i) \cap f(j)} & =\frac{1}{C_{n}^{k}} \sum_{j=1}^{C_{n}^{k}} \sum_{x \in f(i) \cap f(j)} \frac{x}{|f(i) \cap f(j)|} \\
& =\frac{1}{k} \sum_{x \in f(j)} x
\end{aligned}
$$

Hence, the $q$-th power mean of all the arithmetic means of the rows is less than or equal to

$$
\left(\frac{1}{C_{n}^{k}} \sum_{j=1}^{C_{n}^{k}}\left(\frac{\sum_{x \in f(j)} x}{k}\right)^{q}\right)^{\frac{1}{q}}=\left(\frac{1}{C_{n}^{k}} \sum_{\substack{|A|=k \\ A \subset X}}\left(a_{1 A}\right)^{q}\right)^{\frac{1}{q}} .
$$

Applying Lemma 1, it follows from (11) and (12) that

$$
\left(\frac{1}{C_{n}^{k}} \sum_{\substack{|A|=k \\ A \subset X}}\left(a_{1 A}\right)^{q}\right)^{\frac{1}{q}} \geq \frac{1}{C_{n}^{k}} \sum_{\substack{|A|=k \\ A \subset X}} a_{q A} .
$$

Thus, (2) is true for $p=1$. 


\section{REFERENCES}

[1] E. F. Beckenbach and R. Bellman, Inequalities, Ergebnisse der Mathematik und ihrer Grenzgebiete, Band 30, Springer-Verlag, Berlin, 1961. MR 28:1266

[2] B. C. Carlson, R. K. Meany, and S. A. Nelson, An inequality of mixed arithmetic and geometric means, SIAM Review 13(2) (1971), 253-255.

[3] H. Z. Chuan, Note on the inequality of the arithmetic and geometric means, Pacific J. Math. 143(1) (1990), 43-46. MR 91b:26024

[4] G. H. Hardy, J. E. Littlewood, and G. Pólya, Inequalities, second edition, Cambridge University Press, Cambridge, 1952. MR 13:727e

[5] H. Kober, On the arithmetic and geometric means and on Hölder's inequality, Proc. Amer. Math. Soc. 9 (1958), 452-459. MR 20:88

[6] D. S. Mitrinović, Analytic inequalities, Springer-Verlag, New York, 1970. MR 43:448

[7] D. S. Mitrinović, J. E. Pečarić, and A. M. Fink, Classical and New Inequalities in Analysis, Kluwer, Dordrecht, 1993. MR 94c:00004

Department of Mathematics, Shanghai University, Shanghai, 200436, People's RepubLIC OF CHINA

E-mail address: gleng@mail.shu.edu.cn

Department of Mathematics, Shanghai University, Shanghai, 200436, People's RepubLIC OF CHINA

E-mail address: silin_mail@sohu.com

School of Mathematical Sciences, Peking University, Beijing 100871, People's RePUBLIC OF CHINA 